\title{
Fusion of conformal defects in four dimensions
}

\author{
Alexander Söderberg \\ Department of Physics and Astronomy, Uppsala University, \\ Box 516, SE-751 20 Uppsala, Sweden \\ E-mail: alexander.soderberg@physics.uu.se
}

ABSTRACT: We consider two conformal defects close to each other in a free theory, and study what happens as the distance between them goes to zero. This limit is the same as zooming out, and the two defects have fused to another defect. As we zoom in we find a non-conformal effective action for the fused defect. Among other things this means that we cannot in general decompose the two-point correlator of two defects in terms of other conformal defects. We prove the fusion using the path integral formalism by treating the defects as sources for a scalar in the bulk.

Keywords: Conformal Field Theory, Wilson, 't Hooft and Polyakov loops, Effective Field Theories, Conformal and W Symmetry

ARXiv EPrint: 2102.00718 


\section{Contents}

1 Introduction 1

2 Fusion of two Wilson lines, seen from defect correlators 3

$\begin{array}{lll}3 & \text { Proof of fusion through path integral formalism } & 7\end{array}$

4 Fusion of two non-concentric Wilson loops $\quad 8$

5 Fusion of two concentric Wilson loops $\quad 9$

6 Conclusion 11

A Conformal block decomposition $\quad 12$

\section{Introduction}

Recently there have been a lot of research focused on higher dimensional (in dimensions greater than two) conformal field theories (CFT's) in the presence of a boundary (see e.g. [1-4]) or a defect (see e.g. [5-8]). However, the literature regarding higher dimensional CFT's in the presence of several defects is scarce [9-12]. We add to this literature this work where we consider two scalar Wilson lines in a free theory in four dimensions, and study the limit in which they intersect. This corresponds to a fusion of the two defects.

Fusion of defects have previously been studied in two dimensions [13, 14], in supersymmetric theories [15-17], in gauge-invariant integrable systems $[18],{ }^{1}$ and in topological field theories as well as in conformal nets using fusing categories [19-21]. In this paper, we will provide three different examples of fusing two conformal defects in four dimensional free theories using the path integral formalism. To our knowledge this has never been done before, and since we do not make use of the symmetries, the method we present should work for non-conformal defects as well. The fusion in these three examples is done in the same way using the following method:

1. Find the two-point function $\left\langle D_{1} D_{2}\right\rangle$ for the two defects. This should describe a nonperturbative Casimir effect between the defects, and will be given by the exponential of an integral over a two-point function of fields on each defect.

2. Probe this correlator with a bulk field, $\left\langle D_{1} D_{2} \phi\right\rangle$, and study the fusion limit where the distance between the defects go to zero. This will be given by integrals over a two-point function of $\phi$ and a field from one of the defects.

\footnotetext{
${ }^{1}$ AS thanks Simon Ekhammar for pointing out this reference.
} 
3. Find an effective action for the fused defect. The difficulty here lies in identifying what kind of operators appear on the fused defect. In the examples we study, they are directional derivatives w.r.t. the distance vector between the two defects. The fused defect should satisfy

$$
\left\langle D_{1} D_{2} \phi\right\rangle=\left\langle D_{1} D_{2}\right\rangle\left\langle D_{f} \phi\right\rangle
$$

4. Prove the fusion using the path integral formalism by treating the defects as sources for an operator in the bulk. ${ }^{2}$

A higher dimensional CFT enjoy a $\mathrm{SO}(d+1,1)$-symmetry in Euclidean space. We say that a flat (or spherical) $p$-dimensional defect is conformal if a field localized on it satisfy a $\mathrm{SO}(p+1,1) \times \mathrm{SO}(d-p)$-symmetry, where $\mathrm{SO}(p+1,1)$ is the conformal symmetry along the defect, and $\mathrm{SO}(d-p)$ is the group of rotations around the defect. The defect itself, as a $p$-dimensional operator, satisfy $\mathrm{SO}(d-p)$-symmetry.

Correlators with two conformal defects have previously been decomposed in conformal blocks corresponding to local operators in the bulk [9, 12]. Only this block decomposition is known. It is therefore interesting to study whether two defects can be decomposed in another way, which will yield a bootstrap equation for the defect correlators. One might believe that another block decomposition will be in terms of other conformal defects. As we study explicit examples of fusion of conformal defects, we find that the fused defect is not conformal and thus in general there will not be a second decomposition in terms of conformal defects.

This means that in the examples we study we can effectively describe a CFT with two conformal defects as a theory with one non-conformal defect. The latter description may be simpler to study as we only have one defect, although it comes at the cost of losing conformal symmetry along the defect.

The first example we consider is two parallel Wilson lines $D_{ \pm}$separated by a distance $2 R$ in a free theory in four dimensions. ${ }^{3}$ These are one-dimensional defects that does not carry any $\mathrm{SO}(3)$-spin

$$
D_{ \pm}=\exp \left(\lambda_{ \pm} \int_{\mathbb{R}} d x \phi\left(x \hat{x}_{\|} \pm R \hat{x}_{\perp}^{1}\right)\right), \quad \lambda_{ \pm} \in \mathbb{C} .
$$

Here $\hat{x}_{\|}$is the unit vector parallel to the defect, and $\hat{x}_{\perp}^{1}$ is one of the three unit vector orthogonal to the defect. In section 2 we study the three-point correlator $\left\langle D_{+} D_{-} \phi\right\rangle$ between the two defects probed with a bulk field $\phi$, and note that it is the same as a two-point correlator $\left\langle D_{f} \phi\right\rangle$ between another defect and the same bulk field (times a non-perturbative Casimir effect from $\left\langle D_{+} D_{-}\right\rangle$between the two defects). From this we deduce that the new defect $D_{f}$ is a fusion of the other two other defects. We find it to be given by

$$
D_{f}=\exp \left(\sum_{n \geq 0} \frac{\lambda_{-}^{n}+(-1)^{n} \lambda_{+}^{n}}{n !} R^{n} \int_{\mathbb{R}} d x \partial_{x_{\perp}^{1}}^{n} \phi(x)\right) .
$$

\footnotetext{
${ }^{2}$ This might be more complicated for composite operators. For such operators we also have to be careful since they will generate a renormalization group (RG) flow on the defects.

${ }^{3}$ The distance parameter $R$ should be treated as a scale of the theory.
} 
As the distance $R$ between the two defects $D_{ \pm}$goes to zero, the defect $D_{f}$ is conformal. However, as we zoom in and pick up perturbations in $R$, we find dimensionfull coupling constants which break the conformal symmetry. ${ }^{4}$ It is thus not possible to decompose the two-point correlator $\left\langle D_{+} D_{-}\right\rangle$in terms of conformal defects.

The $n=1$ term in the fused defect corresponds to the displacement operator, and it tells us that we have translated $D_{ \pm}$by a distance $\mp R$. One might naively believe that this is a general statement of fusion. However, this is not the case as this operator does not appear in the example of fusion studied in section $4 .^{5}$

In section 3 we treat $D_{ \pm}$and $D_{f}$ as sources for $\phi$. We are then able to show that the path integral is the same regardless of whether we use $D_{+}$and $D_{-}$as a source, or $D_{f}$. This proves the fusion.

In section 4 and 5 we study two other examples of fusion in free theories. Here we consider two scalar Wilson loops (non-concentric and concentric) as opposed to Wilson lines. The defects are fused in a similar manner, and we can again prove it using the path integral formalism. Our main results in the paper are the fusions at equations (2.20), (4.4) and (5.4).

In appendix A we study the block decomposition of the two-point correlators of the circular defects considered in section 4 and 5. This is done using the method of [9]. We find that all of the cross-ratios can be expressed in terms of each other, which means that this block decomposition does not need to be unique.

We conclude in section 6 with some future aspects. Knowing the exponential form of two defects, we can possibly fuse them using the method presented in this paper.

\section{Fusion of two Wilson lines, seen from defect correlators}

We can write defects as exponentials, and one of the simplest examples is a scalar Wilson line in $d=4$ dimensions [5, 22]

$$
D=\exp \left(\lambda \int_{\mathbb{R}} d x \phi(x)\right), \quad \Delta_{\phi}=1 .
$$

Here $\lambda \in \mathbb{C}$ is a dimensionless constant. Using Wick's theorem we find

$$
\begin{aligned}
\langle D\rangle & =1+\sum_{n \geq 1} \frac{\lambda^{n}}{n !} \int_{\mathbb{R}} d x_{1} \ldots \int_{\mathbb{R}} d x_{n}\left\langle\phi\left(x_{1}\right) \ldots \phi\left(x_{n}\right)\right\rangle \\
& =\sum_{n \geq 0} \frac{\lambda^{2 n}}{2^{n} n !}\left(\int_{\mathbb{R}} d x_{1} \int_{\mathbb{R}} d x_{2}\left\langle\phi\left(x_{1}\right) \phi\left(x_{2}\right)\right\rangle\right)^{n} \\
& =\exp \left(\frac{\lambda^{2}}{2} \int_{\mathbb{R}} d x_{1} \int_{\mathbb{R}} d x_{2}\left\langle\phi\left(x_{1}\right) \phi\left(x_{2}\right)\right\rangle\right) .
\end{aligned}
$$

Correlators between local fields behave in the same way as in a homogenous CFT (without the defects). In the free theory it is given by

$$
\left\langle\phi\left(x_{1}\right) \phi\left(x_{2}\right)\right\rangle=\frac{A_{d}}{\left|x_{1}-x_{2}\right|^{2 \Delta_{\phi}}}, \quad A_{d}=\frac{1}{(d-2) S_{d}}=\frac{1}{4 \pi^{2}} .
$$

\footnotetext{
${ }^{4}$ If we do not take into account terms proportional to $R$ we will lose this scale of the theory when fusing the defects.

${ }^{5}$ The author is grateful to Lorenzo Bianchi for a discussion on this.
} 


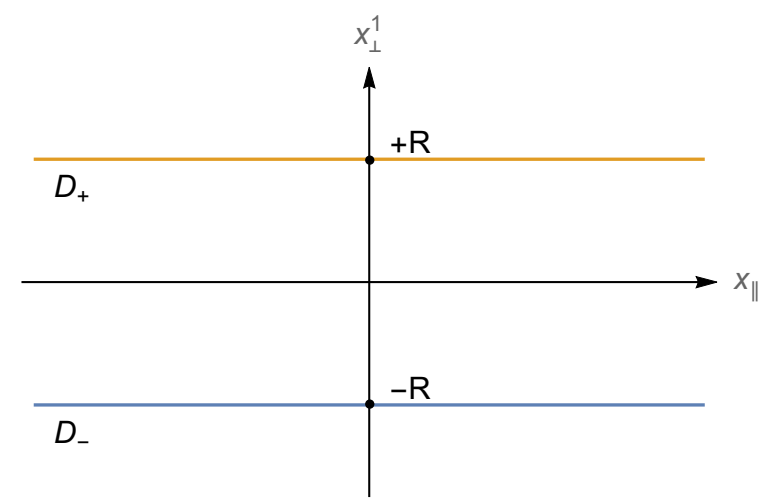

Figure 1. The two line defects are separated by a distance of $2 R$.

Here $S_{d}$ is the area of a $(d-1)$-dimensional sphere, and $\Gamma_{x} \equiv \Gamma(x)$ is the Gamma-function. Integrals over fields on the same defect are divergent, so it is convenient to normalize the propagators by dividing with the one-point functions of the defects

$$
\langle D\rangle_{N} \equiv \frac{\langle D\rangle}{\langle D\rangle}=1
$$

Now place two defects with a distance $2 R>0$ from eachother (see figure 1 )

$$
D_{ \pm}=\exp \left(\lambda_{ \pm} \int_{\mathbb{R}} d x \phi\left(x \hat{x}_{\|} \pm R \hat{x}_{\perp}^{1}\right)\right) \equiv \exp \left(\lambda_{ \pm} \int_{\mathbb{R}} d x \phi_{ \pm}(x)\right), \quad \lambda_{ \pm} \in \mathbb{C} .
$$

Here $\hat{x}_{\|}$is the unit vector along the defect, and $\hat{x}_{\perp}^{1}$ is one of the three unit vectors orthogonal to the defects. It will be convenient for us to use different notations depending on whether we integrate over fields on the same defect, or fields from different defects

$$
I_{ \pm} \equiv \int_{\mathbb{R}} d x \int_{\mathbb{R}} d y\left\langle\phi_{ \pm}(x) \phi_{ \pm}(y)\right\rangle, \quad J \equiv \int_{\mathbb{R}} d x \int_{\mathbb{R}} d y\left\langle\phi_{+}(x) \phi_{-}(y)\right\rangle .
$$

A diagrammatic representation of these integrals are in figure 2. The two-point function is given by

$$
\begin{aligned}
\left\langle D_{+} D_{-}\right\rangle & =\exp \left(\int_{\mathbb{R}} d x\left[\lambda_{+} \phi_{+}(x)+\lambda_{-} \phi_{-}(x)\right]\right) \\
& =\sum_{n \geq 0} \frac{1}{n !}\left\langle\left(\int_{\mathbb{R}} d x\left[\lambda_{+} \phi_{+}(x)+\lambda_{-} \phi_{-}(x)\right]\right)^{n}\right\rangle \\
& =\sum_{n \geq 0} \frac{\left(\lambda_{+}^{2} I_{+}+\lambda_{-}^{2} I_{-}+2 \lambda_{+} \lambda_{-} J\right)^{n}}{2^{n} n !}=\exp \left(\frac{\lambda_{+}^{2} I_{+}+\lambda_{-}^{2} I_{-}}{2}+\lambda_{+} \lambda_{-} J\right) .
\end{aligned}
$$

This yields the normalized correlator

$$
\left\langle D_{+} D_{-}\right\rangle_{N} \equiv \frac{\left\langle D_{+} D_{-}\right\rangle}{\left\langle D_{+}\right\rangle\left\langle D_{-}\right\rangle}=e^{\lambda_{+} \lambda_{-} J}
$$


The $J$-integral is found using a Julian-Schwinger parametrization and regularizing one of the defects such that it is of finite length $2 L \gg 1$

$$
\begin{aligned}
J & =\lim _{L \rightarrow \infty} \int_{-L}^{+L} d y \int_{\mathbb{R}} d x \int_{0}^{\infty} d u \frac{e^{-u(x-y)^{2}-4 u R^{2}}}{4 \pi^{2}}=\lim _{L \rightarrow \infty} \int_{-L}^{+L} d y \int_{0}^{\infty} d u \frac{e^{-4 u R^{2}}}{4 \pi^{3 / 2} \sqrt{u}} \\
& =\lim _{L \rightarrow \infty} \frac{L}{4 \pi R} .
\end{aligned}
$$

This yields

$$
\left\langle D_{+} D_{-}\right\rangle_{N}=\lim _{L \rightarrow \infty} e^{\lambda_{+} \lambda_{-} L /(4 \pi R)} .
$$

The non-perturbative dependence in $R$ describes a Casimir effect between the two defects.

Let us now probe the two-point correlator with a bulk scalar placed at

$$
z \equiv z_{\|} \hat{x}_{\|}+z_{\perp} \hat{x}_{\perp}^{1}, \quad\left|z_{\perp}\right|>R .
$$

Note that in the fusion limit $R \rightarrow 0^{+}$this scalar is not squeezed in between the two defects.

$$
\begin{aligned}
\left\langle D_{+} D_{-} \phi(z)\right\rangle= & \sum_{n \geq 0} \frac{1}{n !}\left\langle\left(\int_{\mathbb{R}} d x\left[\lambda_{+} \phi_{+}(x)+\lambda_{-} \phi_{-}(x)\right]\right)^{n} \phi(z)\right\rangle \\
= & \sum_{n \geq 0} \frac{2 n+1}{(2 n+1) !}\left\langle\left(\int_{\mathbb{R}} d x\left[\lambda_{+} \phi_{+}(x)+\lambda_{-} \phi_{-}(x)\right]\right)^{2 n}\right\rangle \\
& \times\left\langle\int_{\mathbb{R}} d x\left[\lambda_{+} \phi_{+}(x)+\lambda_{-} \phi_{-}(x)\right] \phi(z)\right\rangle \\
= & \left\langle D_{+} D_{-}\right\rangle\left[\lambda_{+} K_{+}(z)+\lambda_{-} K_{-}(z)\right], \\
K_{ \pm}(z) \equiv & \int_{\mathbb{R}} d x\left\langle\phi_{ \pm}(x) \phi(z)\right\rangle .
\end{aligned}
$$

A diagrammatic representation of this integral is in figure 2. This integral can again be solved with a Julian-Schwinger parametrization

$$
K_{ \pm}(z)=\int_{\mathbb{R}} d x \int_{0}^{\infty} d u \frac{e^{-u\left(z_{\|}-x^{2}\right)-u\left(z_{\perp} \mp R\right)^{2}}}{4 \pi^{2}}=\frac{1}{4 \pi\left|z_{\perp} \mp R\right|}=\sum_{n \geq 0} \frac{( \pm R)^{n}}{4 \pi z_{\perp}^{n+1}} .
$$

It yields the normalized correlator ${ }^{6}$

$$
\left\langle D_{+} D_{-} \phi(z)\right\rangle_{N} \equiv \frac{\left\langle D_{+} D_{-} \phi(z)\right\rangle}{\left\langle D_{+}\right\rangle\left\langle D_{-}\right\rangle}=\lim _{L \rightarrow \infty} e^{\lambda_{+} \lambda_{-} L /(4 \pi R)} \sum_{n \geq 0} \frac{\lambda_{+}+(-1)^{n} \lambda_{-}}{4 \pi z_{\perp}^{n+1}} R^{n} .
$$

Now we wish to study whether this correlator can be written in terms of a fused defect

$$
\left\langle D_{+} D_{-} \phi(z)\right\rangle_{N} \stackrel{?}{=}\left\langle D_{+} D_{-}\right\rangle_{N}\left\langle D_{f} \phi(z)\right\rangle_{N}
$$

In order to understand this we need to find some kind of operators that the series in (2.14) will correspond to. For this purpose, let us consider the following defect

$$
D_{n}=\exp \left(\int_{\mathbb{R}} d x \partial_{\perp}^{n} \phi\left(x \hat{x}_{\|}\right)\right), \quad \partial_{\perp} \equiv \partial_{x_{\perp}^{1}}=\partial_{R} .
$$

\footnotetext{
${ }^{6}$ Please note that we can instead choose a normalization where we divide with $\left\langle D_{+} D_{-}\right\rangle$rather than $\left\langle D_{+}\right\rangle\left\langle D_{-}\right\rangle$. Such normalization will remove the non-perturbative Casimir effect.
} 


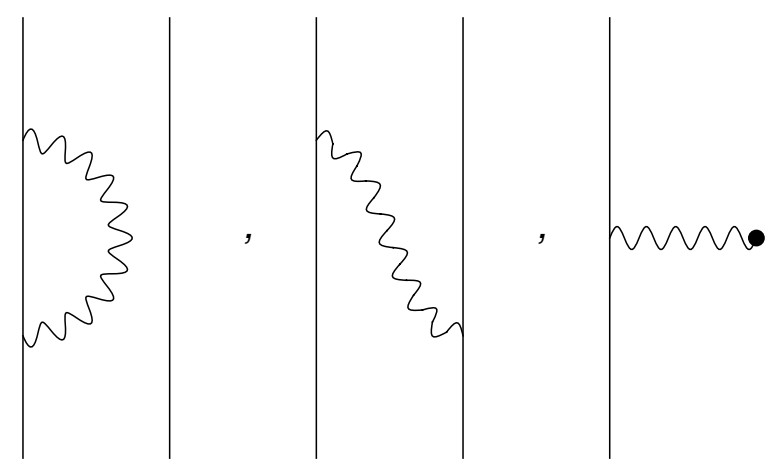

Figure 2. A diagrammatic depiction of the integrals $I_{ \pm}, J$ and $K$.

If we probe its correlator with a bulk scalar ${ }^{7}$

$$
\left\langle D_{n} \phi(z)\right\rangle=\left\langle D_{n}\right\rangle \int_{\mathbb{R}} d x\left\langle\partial_{\perp}^{n} \phi\left(x \hat{x}_{\|}\right) \phi(z)\right\rangle=\left\langle D_{n}\right\rangle \lim _{R \rightarrow 0^{+}} \partial_{\perp}^{n} K_{-}(z)=\left\langle D_{n}\right\rangle \frac{(-1)^{n} n !}{4 \pi z_{\perp}^{n+1}} .
$$

The normalized correlator is thus

$$
\left\langle D_{n} \phi(z)\right\rangle_{N} \equiv \frac{\left\langle D_{n} \phi(z)\right\rangle}{\left\langle D_{n}\right\rangle}=\frac{(-1)^{n} n !}{4 \pi z_{\perp}^{n+1}} \Rightarrow \frac{1}{4 \pi z_{\perp}^{n+1}}=\frac{(-1)^{n}}{n !}\left\langle D_{n} \phi(z)\right\rangle_{N} .
$$

Compare with (2.14) to find

$$
\left\langle D_{+} D_{-} \phi(z)\right\rangle_{N}=\lim _{L \rightarrow \infty} e^{\lambda_{+} \lambda_{-} L /(4 \pi R)} \sum_{n \geq 0} \frac{\lambda_{-}+(-1)^{n} \lambda_{+}}{n !} R^{n}\left\langle D_{n} \phi(z)\right\rangle_{N} .
$$

From this we can deduce that the defects have fused into a single defect

$$
\begin{aligned}
D_{+} D_{-} & =\lim _{L \rightarrow \infty} e^{\lambda_{+} \lambda_{-} L /(4 \pi R)} D_{f}, \\
D_{f} & =\exp \left(\sum_{n \geq 0} \frac{\lambda_{-}+(-1)^{n} \lambda_{+}}{n !} R^{n} \int_{\mathbb{R}} d x \partial_{\perp}^{n} \phi\left(x \hat{x}_{\|}\right)\right) .
\end{aligned}
$$

It is possible to check that this fusion holds for other probed bulk fields, e.g.

$$
\left\langle D_{+} D_{-} \phi^{2}(z)\right\rangle_{N}=\left\langle D_{+} D_{-}\right\rangle_{N}\left\langle D_{f} \phi^{2}(z)\right\rangle_{N} .
$$

Now let us discuss the fused defect at (2.20). In the strict fusing limit it is conformal, but as we zoom in and pick up perturbations in $R$, it has dimensionfull coupling constants, $R^{n}$, and is thus no longer conformal. ${ }^{8}$ Among other things this means that it is not possible for us to use this fusion for bootstrap purposes.

We also notice that the $n=1$ term in the fusion (2.20) corresponds to the displacement operator, which translates $D_{ \pm}$by a distance $\mp R$ in the $\hat{x}_{\perp}^{1}$-direction. One might believe that this is a general feature of fusion, but later in the paper we will see that this is not always the case.

\footnotetext{
${ }^{7}$ In principle we can instead consider $\lim _{R \rightarrow 0^{+}} \partial_{\perp}^{n} K_{+}$. However, as we will see in the next section, this yields the wrong fusion.

${ }^{8}$ The terms proportional to $R$ should be included in the description of the fused defect to make sure we are not losing this scale of the theory.
} 


\section{Proof of fusion through path integral formalism}

In order to prove that the fusion (2.20) is correct we need to show that it does not matter for the path integral (which generates all of the correlators) whether we consider the two line defects, or the fused defect. The path integral is

$$
Z[J]=\int \mathcal{D} \phi \exp \left[\int_{\mathbb{R}^{d}} d^{d} x\left(\frac{(\partial \phi)^{2}}{2}+J \phi\right)\right] .
$$

Let us perform a partial integration on the source term and add a zero on the form $\left(\partial^{-1} J\right)^{2}-\left(\partial^{-1} J\right)^{2}$. Then we complete the square for $\phi$ and perform a partial integration on the residual $\left(\partial^{-1} J\right)^{2}$-term

$$
\begin{aligned}
Z[J] & =\int \mathcal{D} \phi \exp \left[\int_{\mathbb{R}^{d}} d^{d} x\left(\frac{(\partial \phi)^{2}-2 \partial^{-1} J \partial \phi+\left(\partial^{-1} J\right)^{2}-\left(\partial^{-1} J\right)^{2}}{2}\right)\right] \\
& =\int \mathcal{D} \phi \exp \left[\int_{\mathbb{R}^{d}} d^{d} x\left(\frac{\left[\partial\left(\phi-\partial^{-2} J\right)\right]^{2}+J \partial^{-2} J}{2}\right)\right]
\end{aligned}
$$

Here $\partial^{-2}$ is the Green's function $G$. Perform the field redefinition

$$
\phi(x) \rightarrow \phi(x)+\left(\partial^{-2} J\right)(x) \equiv \phi(x)+\int_{\mathbb{R}^{d}} d^{d} y G(x-y) J(y) .
$$

This gives us the normalized path integral

$$
\frac{Z[J]}{Z[0]}=e^{\zeta[J]}, \quad \zeta[J]=\int_{\mathbb{R}^{d}} d^{d} x \int_{\mathbb{R}^{d}} d^{d} y \frac{J(x) G(x-y) J(y)}{2} .
$$

To prove that the fusion (2.20) is correct we need to show that the above path integral for the two line defects is the same as that for the fused defect. We will do this by writing the defects as sources. For the two defects we write

$$
J_{b}(x)=\lambda_{+} \delta\left(\vec{x}_{\perp}-\vec{R}\right)+\lambda_{-} \delta\left(\vec{x}_{\perp}+\vec{R}\right)+\tilde{J}(x), \quad \vec{R}=R \hat{x}_{\perp}^{1} .
$$

Here $\vec{x}_{\perp}$ is the vector orthogonal to the defects, and $\tilde{J}(x)$ is the actual source for $\phi$. For the fused defect we write

$$
J_{f}(x)=\delta\left(\vec{x}_{\perp}\right) \sum_{n \geq 0} \frac{\lambda_{-}+(-1)^{n} \lambda_{+}}{n !} R^{n} \partial_{x_{\perp}^{1}}^{n}+\tilde{J}(x) .
$$

We want to show that

$$
\zeta\left[J_{b}\right]=\zeta\left[J_{f}\right] .
$$

If we insert (3.5) into (3.4)

$$
\begin{aligned}
\zeta\left[J_{b}\right]= & \int_{\mathbb{R}} d x_{\|} \int_{\mathbb{R}} d y_{\|}\left(\frac{\lambda_{+}^{2}+\lambda_{-}^{2}}{2} G\left(\vec{s}_{\|}\right)+\lambda_{+} \lambda_{-} G\left(\vec{s}_{\|}+2 \vec{R}\right)\right)+ \\
& +\int_{\mathbb{R}} d x_{\|} \int_{\mathbb{R}^{d}} d^{d} y \tilde{J}(y)\left[\lambda_{+} G\left(\vec{s}_{\|}+\vec{y}_{\perp}-\vec{R}\right)+\lambda_{-} G\left(\vec{s}_{\|}+\vec{y}_{\perp}+\vec{R}\right)\right]+ \\
& +\int_{\mathbb{R}^{d}} d^{d} x \int_{\mathbb{R}^{d}} d^{d} y \frac{\tilde{J}(x) G(x-y) \tilde{J}(y)}{2}
\end{aligned}
$$


Here $\vec{x}_{\perp}, \vec{y}_{\perp}$ are vectors orthogonal to the defects, and $\vec{s}_{\|}$is the difference between the parallel coordinates along the defects

$$
\vec{s}_{\|} \equiv\left(x_{\|}-y_{\|}\right) \hat{x}_{\|}, \quad \vec{y}_{\perp}=y_{i} \hat{x}_{\perp}^{i}, \quad \vec{x}_{\perp}=x_{i} \hat{x}_{\perp}^{i}, \quad i \in\{1,2,3\} .
$$

We want to show that the functional (3.8) is the same as (3.6) inserted in (3.4)

$$
\begin{aligned}
\zeta\left[J_{f}\right]= & \int_{\mathbb{R}} d x_{\|} \int_{\mathbb{R}} d y_{\|} \sum_{m, n \geq 0} \frac{\lambda_{+}^{2} R^{m+n}+\lambda_{-}^{2}(-R)^{m+n}+2 \lambda_{+} \lambda_{-}(-R)^{m} R^{n}}{2 m ! n !} \\
& \times\left.\partial_{x_{\perp}^{1}}^{m} \partial_{y_{\perp}^{1}}^{n} G\left(\vec{s}_{\|}+\vec{x}_{\perp}-\vec{y}_{\perp}\right)\right|_{\vec{x}_{\perp}=\vec{y}_{\perp}=0}+ \\
& +\left.\int_{\mathbb{R}} d x \int_{\mathbb{R}^{d}} d^{d} y \tilde{J}(y) \sum_{n \geq 0} \frac{\lambda_{-} R^{n}+\lambda_{+}(-R)^{n}}{n !} \partial_{x_{\perp}^{1}}^{n} G\left(\vec{s}_{\|}+\vec{x}_{\perp}-\vec{y}_{\perp}\right)\right|_{\vec{x}_{\perp}=0}+ \\
& +\int_{\mathbb{R}^{d}} d^{d} x \int_{\mathbb{R}^{d}} d^{d} y \frac{\tilde{J}(x) G(x-y) \tilde{J}(y)}{2}
\end{aligned}
$$

This is the Taylor expansion of (3.8) around $R=0$. Here we used that the Green's function is symmetric w.r.t. $x$ and $y$

$$
G(x-y)=G(y-x) \quad \Rightarrow \quad G\left(\vec{s}_{\|}, \vec{x}_{\perp}-\vec{y}_{\perp}\right)=G\left(\vec{s}_{\|}, \vec{y}_{\perp}-\vec{x}_{\perp}\right) .
$$

This proves that the defect in (2.20) is indeed the fusion of the line defects in (2.5).

\section{Fusion of two non-concentric Wilson loops}

In this section we will provide another example of fusion in a free theory. We will consider two scalar Wilson loops of radius $r$ in $d=4$ dimensions at a distance $2 R \equiv 2|\vec{R}|>0$ from each other (see figure 3 )

$$
D_{ \pm}=\exp \left(\lambda_{ \pm} \int_{0}^{2 \pi} d \theta \phi\left(r\left(c_{\theta} \hat{x}_{\|}^{1}+s_{\theta} \hat{x}_{\|}^{2}\right) \pm \vec{R}\right)\right) \equiv \exp \left(\lambda_{ \pm} \int_{0}^{2 \pi} d \theta \phi_{ \pm}(\theta)\right) .
$$

Here $c_{\theta} \equiv \cos (\theta), s_{\theta} \equiv \sin (\theta)$ and $\hat{x}_{\|}^{j}, j \in\{1,2\}$ are two of the four unit vectors. $\vec{R}$ is a vector orthogonal to both $\hat{x}_{\|}^{j}$. We can proceed in the same way as in section 2, encountering slightly different integrals corresponding to equation (2.6) and (2.12) (we probe the defect correlator with a bulk field at origo)

$$
\begin{aligned}
J & =\int_{0}^{2 \pi} d \theta_{1} \int_{0}^{2 \pi} d \theta_{2}\left\langle\phi_{+}\left(\theta_{1}\right) \phi_{-}\left(\theta_{2}\right)\right\rangle \\
& =\frac{A_{d}}{2^{\Delta_{\phi}}} \int_{0}^{2 \pi} d \theta_{1} \int_{0}^{2 \pi} d \theta_{2} \int_{0}^{\infty} d u \frac{u^{\Delta_{\phi}-1}}{\Gamma_{\Delta_{\phi}}} e^{-u\left(r^{2}\left(1-c_{\theta_{1}-\theta_{2}}\right)+2 R^{2}\right)} \\
& =\frac{\pi^{2} A_{d}}{2^{\Delta_{\phi}-2}\left(r^{2}+2 R^{2}\right)^{\Delta_{\phi}}}{ }^{2} F_{1}\left(\frac{\Delta_{\phi}}{2}, \frac{\Delta_{\phi}+1}{2} ; 1 ; \frac{r^{4}}{\left(r^{2}+2 R^{2}\right)^{2}}\right)=\frac{1}{4 R \sqrt{r^{2}+R^{2}}} \\
K_{ \pm} & =\int_{0}^{2 \pi} d \theta\left\langle\phi_{ \pm}(\theta) \phi(0)\right\rangle=\int_{0}^{2 \pi} d \theta \frac{A_{d}}{\left(r^{2}+R^{2}\right)^{\Delta_{\phi}}}=\frac{1}{2 \pi\left(r^{2}+R^{2}\right)} \\
& =\sum_{n \geq 0} \frac{(-1)^{n} R^{2 n}}{2 \pi r^{2(n-1)}}
\end{aligned}
$$




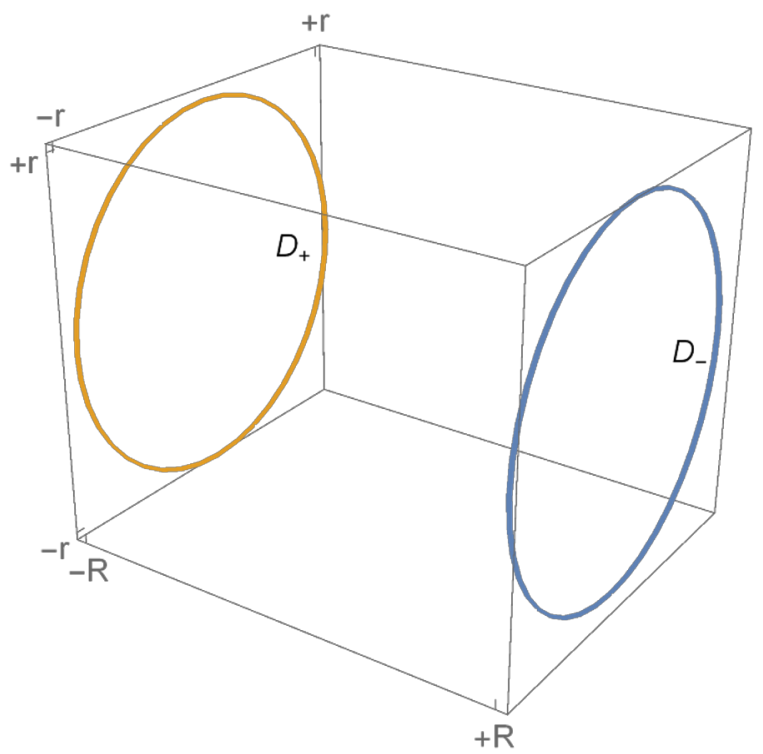

Figure 3. Two circular defects that are separated by a distance of $2 R$.

We find the fusing to be

$$
\begin{aligned}
D_{+} D_{-} & =e^{\lambda_{+} \lambda_{-} /\left(4 R \sqrt{r^{2}+R^{2}}\right)} D_{f}, \\
D_{f} & =\exp \left(\sum_{n \geq 0} \frac{\lambda_{+}+\lambda_{-}}{(2 n) !} R^{2 n} \int_{0}^{2 \pi} d \theta \nabla_{R}^{2 n} \phi\left(r\left(c_{\theta} \hat{x}_{\|}^{1}+s_{\theta} \hat{x}_{\|}^{2}\right)\right)\right) .
\end{aligned}
$$

Here $\nabla_{R} \equiv \vec{R} \cdot \vec{\nabla}$ is the directional derivative w.r.t. the vector $\vec{R}$. The fusion is proven in the same way as in section 3 using the path integral formalism and by treating the defects as sources for the fundamental scalar $\phi$ in the bulk. Unlike the two line defects in section 2, the displacement operator does not appear in the effective action of the fused defect.

\section{Fusion of two concentric Wilson loops}

The last example of fusion that we will study is between two concentric circles. We will again consider two scalar Wilson loops in $d=4$ dimensions in a free theory, separated by a distance $2 R$. Unlike the previous section the defects will be concentric (see figure 4), i.e. the Wilson loops will have different radii $r \pm R$

$$
D_{ \pm}=\exp \left(\lambda_{ \pm} \int_{0}^{2 \pi} d \theta \phi\left((r \pm R)\left(c_{\theta} \hat{x}_{\|}^{1}+s_{\theta} \hat{x}_{\|}^{2}\right)\right)\right) \equiv \exp \left(\lambda_{ \pm} \int_{0}^{2 \pi} d \theta \phi_{ \pm}(\theta)\right)
$$




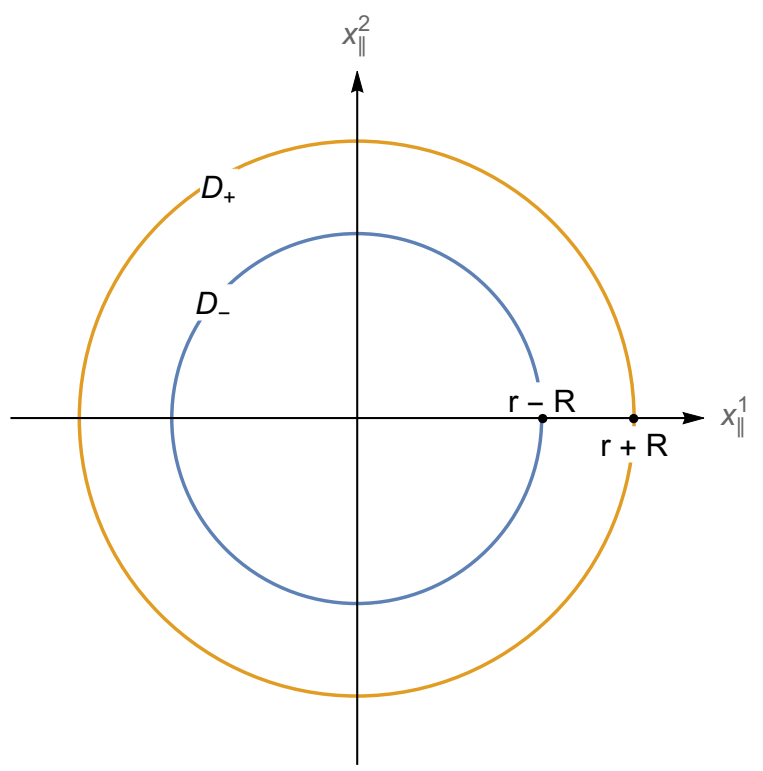

Figure 4. The concentric circular defects are separated by a distance of $2 R$.

The integrals we encounter in $\left\langle D_{+} D_{-}\right\rangle$and $\left\langle D_{+} D_{-} \phi(0)\right\rangle$ (corresponding to equation (2.6) and (2.12)) are given by

$$
\begin{aligned}
J & =\int_{0}^{2 \pi} d \theta_{1} \int_{0}^{2 \pi} d \theta_{2}\left\langle\phi_{+}\left(\theta_{1}\right) \phi_{-}\left(\theta_{2}\right)\right\rangle \\
& =\frac{A_{d}}{2^{\Delta_{\phi}}} \int_{0}^{2 \pi} d \theta_{1} \int_{0}^{2 \pi} d \theta_{2} \int_{0}^{\infty} d u \frac{u^{\Delta_{\phi}-1}}{\Gamma_{\Delta_{\phi}}} e^{-u\left(r^{2}+R^{2}-\left(r^{2}-R^{2}\right) c_{\theta_{1}-\theta_{2}}\right)} \\
& =\frac{\pi^{2} A_{d}}{2^{\Delta_{\phi}-2}\left(r^{2}+2 R^{2}\right)^{\Delta_{\phi}}}{ }^{2} F_{1}\left(\frac{\Delta_{\phi}}{2}, \frac{\Delta_{\phi}+1}{2} ; 1 ; \frac{\left(r^{2}-R^{2}\right)^{2}}{\left(r^{2}+R^{2}\right)^{2}}\right)=\frac{1}{4 \pi R}, \\
K_{ \pm} & =\int_{0}^{2 \pi} d \theta\left\langle\phi_{ \pm}(\theta) \phi(0)\right\rangle=\int_{0}^{2 \pi} d \theta \frac{A_{d}}{(r \pm R)^{2 \Delta_{\phi}}}=\frac{1}{2 \pi(r \pm R)^{2}} \\
& =\sum_{n \geq 0} \frac{(\mp 1)^{n}(n+1) R^{n}}{2 \pi r^{n+2}} .
\end{aligned}
$$

The fusion is very similar to the line defects in section 2

$$
\begin{aligned}
D_{+} D_{-} & =e^{\lambda_{+} \lambda_{-} /(4 \pi R)} D_{f}, \\
D_{f} & =\exp \left(\sum_{n \geq 0} \frac{\lambda_{-}+(-1)^{n} \lambda_{+}}{n !} R^{n} \int_{0}^{2 \pi} d \theta \partial_{R}^{n} \phi\left(r\left(c_{\theta} \hat{x}_{\|}^{1}+s_{\theta} \hat{x}_{\|}^{2}\right)\right)\right) .
\end{aligned}
$$

Here $\partial_{R}^{n}$ are derivatives in the Radial direction. This fusion is proven in the same way as in section 3 , and just like the two line defects in section 2, the $n=1$ term in this fusion corresponds to the displacement operator. 


\section{Conclusion}

We have shown how several different defects in Gaussian models can be fused using one and the same method. Since we are not making use of the conformal symmetry along the defects, this procedure should work for non-conformal defects as well. Although we need to be able to express the defects as exponentials.

It would be interesting to study whether this method works for fusion of defects with composite operators or in an interacting theory (say with a quartic interaction in the bulk). One has to be careful though, as this will generate an RG flow on the defects. This will affect the Wick contractions in equations (2.2), (2.7) and the equation above (2.12). In the examples that we considered, it might generate an RG flow for the constants $\lambda_{ \pm}$. The defects we consider breaks the $O(N)$-symmetry of the bulk scalars. This means that the RG flow might be similar to that in the extraordinary phase transition near a boundary $[23,24] .{ }^{9}$

We can also proceed to study fusion of higher dimensional defects. This could possibly connect the method from this paper with gluing of quantum field theories discussed in [25]. Here it is important that the two defects are parallel to each other and of the same dimensions. I.e. it is not clear what the fusion of a $p$ - and a $q$-dimensional defect (with $p>q$ ) would yield. It might give us a $p$-dimensional defect with a $q$-dimensional defect on top of it. This would not be particularly useful as the theory still contain two defects after the fusion, just on top of each other instead of next to each other.

It is also worthwhile to study whether fusion of conformal defects can be realized using symmetry arguments. This can yield a general statement of the operators that appear on the effective action for the fused defect. It might give us a clue as to why the displacement operator did not appear in the fusion (4.4), while it did in the other two examples.

Let us also comment a bit on the conformal block decomposition in [9]. The defect twopoint correlators in section 4 and 5 can in principle be decomposed in these blocks using this method, and there should be three number of independent cross-ratios. However, we find in appendix A that these cross-ratios are not independent from each other for two parallel circles of codiemension three in four spacetime dimensions. ${ }^{10}$ This means that we cannot guarantee that this conformal block decomposition is unique. ${ }^{11}$

In the case of concentric Wilson loops studied in section 5, we can also (in principle) decompose the defect two-point correlator using the method in [12]. However, in order to use this method we first need to find the Harish-Chandra wave-functions for codimension three defects $(N=3)$.

So it seems like we need some more delicate methods in order to decompose the twopoint correlators of defects that are studied in this paper. We also have to understand what to do with the Casimir effect between the two defect when decomposing these correlators in conformal blocks. ${ }^{12}$

\footnotetext{
${ }^{9}$ The author thanks Parijat Dey for pointing this out.

${ }^{10}$ As in figure 3 , but with different radii on the two circles.

${ }^{11}$ I.e. we should treat appendix A as a step closer to an example of this block decomposition. Something that has not been done before to our knowledge.

${ }^{12} \mathrm{AS}$ is grateful to Tobias Hansen and Andrea Manenti for a discussion on this.
} 
It would also be interesting to study whether fusion of defects can be applied to some more concrete physical examples. One such example may be the codimension two Rényi twist defects (RTD's) that appear in the context of Rényi entropy and mutual information [26-28]. The branch cut generated by an RTD yields $n$ replicas of the bulk CFT, and the Renyi entropy as well as the mutual information is found from the expaction value of one and two RTD's respectively. In the $n \rightarrow 1$ limit (which corresponds to a trivial defect with no branch cut), the Renyi entropy gives us the entanglement entropy. Especially interesting for us is the so-called $c$-function in three dimensions (related to the trace anomaly) which can be found through the fusion limit of two RTD's that span concentric circles [29]. It is possible that this can be understood as a fused defect using the method of this paper. Unfortunately though, an exponential form of the RTD is not known.

\section{Acknowledgments}

The author is grateful to Marco Meineri and Emilio Trevisani for discussions regarding this project and for commenting on the manuscript. He is thankful to the organizers of the BOOTSTRAP 2019 workshop at Perimeter institute, where a majority of this project took place. AS expresses his thanks to the people who went to the seminar at Uppsala University where this work was presented, and for the discussions that followed thereafter. This work is supported by Knut and Alice Wallenberg Foundation KAW 2016.0129.

\section{A Conformal block decomposition}

In this appendix we study the conformal block decomposition in [9] of the defect two-point correlators in section 4 and 5. For this purpose we will assume the more general scalar Wilson loop

$$
D_{ \pm}=\exp \left(\lambda_{ \pm} \int_{0}^{2 \pi} d \theta \phi\left(r_{ \pm}\left(c_{\theta} \hat{x}_{\|}^{1}+s_{\theta} \hat{x}_{\|}^{2}\right) \pm \rho \hat{x}_{\perp}^{1}\right)\right) .
$$

Here the two defects are of different radii $r_{+}$and $r_{-}$, and $2 \rho$ is the distance between the defects. We get the configuration in section 4 in the limit $r_{+} \rightarrow r_{-} \equiv r$ with $\rho \hat{x}_{\perp}^{1} \equiv \vec{R}$, and that in section 5 in the limit $\rho \rightarrow 0$ with $r_{ \pm}=r \pm R$. We need the following lightcone vectors to find the conformal cross-ratios

$$
\begin{aligned}
P_{ \pm}^{1} & =(0,0,0,0,1,0), \\
P_{ \pm}^{2} & =(0,0,0,0,0,1), \\
P_{ \pm}^{3} & =\left(\frac{1}{r_{ \pm}}, \frac{\rho^{2}}{r_{ \pm}}-r_{ \pm}, \pm \frac{\rho}{r_{ \pm}}, 0,0,0\right) .
\end{aligned}
$$

We are interested in the matrix

$$
M^{\alpha \beta}=\left(\vec{P}_{+}^{\alpha} \cdot \vec{P}_{-}^{\gamma}\right)\left(\vec{P}_{+}^{\gamma} \cdot \vec{P}_{-}^{\beta}\right)=\operatorname{diag}\left(1,1, \xi^{2}\right)^{\alpha \beta}, \quad \xi=\frac{r_{-}^{2}+r_{+}^{2}-4 \rho^{2}}{2 r_{-} r_{+}}
$$

Here $\alpha, \beta, \gamma \in\{1,2,3\}$, where summation over $\gamma$ is implicit. The three cross-ratios, $\eta_{a}$, with $a \in\{1,2,3\}$, are given by

$$
\eta_{a}=\operatorname{tr}\left(M^{a}\right)=2+\xi^{2 a} .
$$


As we can see, they can all be expressed in terms of another cross-ratio $\xi$, and thus they are not independent from each other. This means that we cannot guarantee that the conformal block decomposition is unique. We can proceed to follow the procedure in [9] to find an ODE (w.r.t. $\xi$ ) for the conformal blocks that we can possibly solve as a series expansion in $\xi$. However, since we cannot determine whether this decomposition is unique, we will not write out any details on this. The interested reader may study the attached Mathematica file in the supplementary material.

Open Access. This article is distributed under the terms of the Creative Commons Attribution License (CC-BY 4.0), which permits any use, distribution and reproduction in any medium, provided the original author(s) and source are credited.

\section{References}

[1] C.P. Herzog and N. Kobayashi, The $O(N)$ model with $\phi^{6}$ potential in $R^{2} \times R^{+}$, JHEP 09 (2020) 126 [arXiv : 2005. 07863] [inSPIRE].

[2] C. Behan, L. Di Pietro, E. Lauria and B.C. Van Rees, Bootstrapping boundary-localized interactions, JHEP 12 (2020) 182 [arXiv:2009.03336] [INSPIRE].

[3] V. Procházka and A. Söderberg, Spontaneous symmetry breaking in free theories with boundary potentials, arXiv:2012.00701 [INSPIRE].

[4] P. Dey and A. Söderberg, On analytic bootstrap for interface and boundary CFT, arXiv:2012.11344 [INSPIRE].

[5] M. Billò, V. Gonçalves, E. Lauria and M. Meineri, Defects in conformal field theory, JHEP 04 (2016) 091 [arXiv: 1601.02883] [INSPIRE].

[6] A. Söderberg, Anomalous dimensions in the $W F O(N)$ model with a monodromy line defect, JHEP 03 (2018) 058 [arXiv:1706.02414] [INSPIRE].

[7] M. Lemos, P. Liendo, M. Meineri and S. Sarkar, Universality at large transverse spin in defect CFT, JHEP 09 (2018) 091 [arXiv:1712.08185] [INSPIRE].

[8] E. Lauria, M. Meineri and E. Trevisani, Spinning operators and defects in conformal field theory, JHEP 08 (2019) 066 [arXiv: 1807.02522] [INSPIRE].

[9] A. Gadde, Conformal constraints on defects, JHEP 01 (2020) 038 [arXiv:1602.06354] [INSPIRE].

[10] M. Fukuda, N. Kobayashi and T. Nishioka, Operator product expansion for conformal defects, JHEP 01 (2018) 013 [arXiv:1710.11165] [INSPIRE].

[11] N. Kobayashi and T. Nishioka, Spinning conformal defects, JHEP 09 (2018) 134 [arXiv: 1805.05967] [INSPIRE].

[12] M. Isachenkov, P. Liendo, Y. Linke and V. Schomerus, Calogero-Sutherland approach to defect blocks, JHEP 10 (2018) 204 [arXiv:1806.09703] [INSPIRE].

[13] C. Bachas and I. Brunner, Fusion of conformal interfaces, JHEP 02 (2008) 085 [arXiv:0712.0076] [INSPIRE].

[14] A. Konechny, Fusion of conformal interfaces and bulk induced boundary RG flows, JHEP 12 (2015) 114 [arXiv: 1509.07787] [INSPIRE]. 
[15] A. Kapustin and N. Saulina, The algebra of Wilson-'t Hooft operators, Nucl. Phys. B 814 (2009) 327 [arXiv: 0710.2097] [INSPIRE].

[16] N. Saulina, A note on Wilson-'t Hooft operators, Nucl. Phys. B 857 (2012) 153 [arXiv:1110.3354] [INSPIRE].

[17] R. Moraru and N. Saulina, OPE of Wilson-'t Hooft operators in $N=4$ and $N=2$ SYM with gauge group $G=\mathrm{PSU}(3)$, arXiv:1206.6896 [INSPIRE].

[18] K. Costello, E. Witten and M. Yamazaki, Gauge theory and integrability, I, ICCM Not. 06 (2018) 46 [arXiv:1709.09993] [INSPIRE].

[19] P. Etingof, D. Nikshych and V. Ostrik, On fusion categories, math.QA/0203060.

[20] A. Bartels, C.L. Douglas and A. Henriques, Conformal nets III: fusion of defects, arXiv: 1310.8263 [INSPIRE].

[21] C.L. Douglas, C. Schommer-Pries and N. Snyder, Dualizable tensor categories, arXiv: 1312.7188 [INSPIRE].

[22] A. Kapustin, Wilson-'t Hooft operators in four-dimensional gauge theories and S-duality, Phys. Rev. D 74 (2006) 025005 [hep-th/0501015] [INSPIRE].

[23] M.A. Shpot, Boundary conformal field theory at the extraordinary transition: the layer susceptibility to $O(\varepsilon)$, JHEP 01 (2021) 055 [arXiv: 1912.03021] [INSPIRE].

[24] P. Dey, T. Hansen and M. Shpot, Operator expansions, layer susceptibility and two-point functions in BCFT, JHEP 12 (2020) 051 [arXiv: 2006.11253] [INSPIRE].

[25] M. Dedushenko, Gluing. Part I. Integrals and symmetries, JHEP 04 (2020) 175 [arXiv: 1807.04274] [INSPIRE].

[26] P. Calabrese and J.L. Cardy, Entanglement entropy and quantum field theory, J. Stat. Mech. 0406 (2004) P06002 [hep-th/0405152] [INSPIRE].

[27] H. Casini and M. Huerta, Entanglement entropy in free quantum field theory, J. Phys. A 42 (2009) 504007 [arXiv: 0905. 2562] [INSPIRE].

[28] B. Chen, Z.-Y. Fan, W.-M. Li and C.-Y. Zhang, Holographic mutual information of two disjoint spheres, JHEP 04 (2018) 113 [arXiv:1712.05131] [INSPIRE].

[29] H. Casini, M. Huerta, R.C. Myers and A. Yale, Mutual information and the F-theorem, JHEP 10 (2015) 003 [arXiv:1506.06195] [INSPIRE]. 